\title{
Forschung im Fluge
}

\author{
Wie UAS die Wissenschaft bereichern und \\ vor neue Herausforderungen stellen
}

Der Einsatz von Unmanned Aerial Systems (UAS) revolutioniert die Beobachtung umweltrelevanter Faktoren. Sie ermöglichen die Vermessung und Kartierung relativ großer, oft schwierig oder gänzlich unzugänglicher Flächen mit hoher räumlicher Auflösung zu einem selbstbestimmten Zeitpunkt, mit überschaubarem Zeitaufwand im Feld und mit unterschiedlichsten Sensoren. Ihr Einsatz eröffnet außerdem die Möglichkeit weitgehend zerstörungs- und rückwirkungsfreier Aufnahmen im Gelände. Die Forschung ist dabei dem rechtlichen und regulatorischen Rahmen verpflichtet, der das Fliegen im öffentlichen Raum ordnet und reguliert, z. B. hinsichtlich einer Kenntnisnachweispflicht für Pilotinnen und Piloten, Einschränkungen der Flugrouten oder Genehmigungsverfahren. Der Einsatz von UAS bietet aber erheblichen Mehrwert: in den Erd- und Umweltwissenschaften, der Agrarforschung, im Monitoring von Naturrisiken, in der Archäologie, der Zoologie, der Sicherheitsforschung und für viele weitere Anwendungen.

\section{Fast-paced research}

Challenges and Opportunities of UAS for Research

Unmanned aerial systems (UAS) currently revolutionize the monitoring, observation, and research opportunities of environmental processes. They provide many positive aspects, such as high spatial and temporal resolution for environmental monitoring and the definition of the observation time. In addition, UAS allow the use of a variety of sensors and instruments and often collect data outside the realm of the optical spectrum - all leading to non-destructive and non-invasive mapping. The utilization of UAS requires a robust legal and regulatory framework that manages the public airspace and ensures that public, commercial, and academic applications are well defined and supported. This includes the requirement of a certificate of knowledge, flight route restrictions due to airspace limitations, and an authorized institution to hand out permits. These legal constraints and regulations are impor-

This is an article distributed under the terms of the Creative Commons Attribution License CCBY 4.0 (https://creativecommons.org/licenses/by/4.0/)

https://doi.org/10.14512/tatup.27.3.45

Submitted: 23.07.2018. Peer reviewed. Accepted: 08.10.2018 tant and provide scientific applications with large potential, for example in the fields of earth and environmental science, agriculture and ecology, the monitoring of natural hazards, archaeology, zoology, and in security research - and many more applications.

Keywords: UAS in research, structure from motion, certificate of knowledge, earth system science, environmental monitoring

\section{Einleitung}

Die unbemannte Luftfahrt hat mit dem Einsatz von UAS (Unmanned Aerial System) in den letzten Jahren riesige Entwicklungsschritte gemacht. Aus einem zivilen Nischensegment für Bastler und Enthusiasten hat sich ein großer eigener Wirtschaftszweig mit professionellen Systemen, Dienstleistungen und Leistungsmerkmalen entwickelt. Experten rechnen weltweit mit weiterem Wachstum und dem Ausbau dieser Branche hin zu ganz neuen Möglichkeiten im Transportwesen (D'Andrea 2014; Thiels et al. 2015) und der wissenschaftlichen Anwendung als Träger von Sensoren für Forschungs- und Entwicklungsprojekte.

Das ursprüngliche Aufgabenfeld - die Aufnahme von Luftbildern - ist mit weit komplexeren Anwendungsszenarien ergänzt worden. Die angewandten Wissenschaften profitieren von den technologischen Entwicklungen aus der Forschung zur Elektromobilität sowie von Entwicklungen, die aus der Luftund Raumfahrt stammen. Anwendungen in der Umweltforschung, der Geographie oder der Biologie sind durch Innovationen im Bereich der Präzisionssteuerung und der Fluglagenkontrolle möglich. UAS erlauben es, große Flächen schnell und effizient zu kartieren, und mit hohen räumlichen Auflösungen zu vermessen. Die zeitliche Selbstbestimmung des Fluges sowie der Zugang zu kaum erreichbaren Gebieten, zum Beispiel im Katastrophenfall oder in extremen Klima- und Topographiebe- 
reichen (Haala und Schwieger 2017), sind Vorzüge, die für den Einsatz von UAS in der Wissenschaft sprechen.

UAS sind dank intelligenter Bus-Systeme in der Lage unterschiedlichste Sensortechniken zu transportieren. Neben rein optischen Aufzeichnungen können multispektrale Vermessungen, die z. B. den Infrarot- oder Ultraviolett-Bereich umfassen, aktive Verfahren wie Radar-, Laser- und Lidar-Messungen oder sogar chemische Sensoren eingesetzt werden. Speziell aufgezeichnete Daten werden durch komplexe Verfahren, wie z. B. dem Structure-from-Motion (SfM) Prozess (Westoby et al. 2012), zu dreidimensionalen Strukturen zusammengeführt und liefern Informationen, die in zahlreiche wissenschaftliche Anwendungen integriert werden. Aufgrund der geringen logistischen Anfor-

\section{Herausforderungen}

Während der Einsatz von UAS in der Wissenschaft neue Wege der Datenaufzeichnung und somit neue Informationen erlaubt, steht die Forschung vor neuen Herausforderungen. Diese sind auf unterschiedlichen Ebenen zu finden:

\section{Dateninfrastrukturen}

Durch die schnelle und flächendeckende Vermessung und Beobachtung werden entsprechend große und komplexe Datenmengen erzeugt. Die Wissenschaft stößt hier bereits jetzt an ihre Grenzen: sowohl im Handling dieser Daten als auch bei ihrer Speicherung, Zugänglichkeit und Verifikation. Die in der Wis-

\section{Autonome Drohnen könnten in Katastrophengebieten eingesetzt werden, um Menschen zu retten oder Informationen für Entscheider zu sammeln.}

derungen an den Einsatz von UAS werden zudem Langzeitstudien möglich, die z. B. saisonale Veränderungen der Biodiversität (Saarinen et al. 2018) oder erosionsbedingte Änderungen der Topographie (Turner et al. 2015) mit bisher unerreichter Auflösung ermöglichen. Archäologen, Landschaftsplaner und Baudenkmalpfleger (Haala und Schwieger 2017) nutzen UAS seit einiger Zeit, um das kulturelle Erbe in Form dreidimensionaler Aufzeichnungen zu bewahren (Fallavollita et al. 2013; Campana 2017) und um selbst kleinste, meist klimabedingte Änderungen bis hinein in niedrige Zentimeter-Skalen (Nebiker et al. 2008; Turner et al. 2012) zu erkennen.

Einen besonderen Aspekt erfährt dies in der Vermessung und Aufzeichnung historischer Großobjekte, die durch Siedlungsdruck oder Klimaveränderungen der Zerstörung ausgeliefert sind. Um größere Zielgebiete strukturiert befliegen zu können, kommt der reine Sichtflug schnell an die Grenzen - UAS müssen im programmierten Betrieb in autonome und teilautonome Flugverfahren überführt werden. Die reine Sichtweite ( $v i$ sual line of sight) ist dann keine Beschränkung mehr und eröffnet ganz neue Einsatzgebiete (Korn und Edinger 2008). Auch aus Gründen der Flugsicherung sind autonome Systeme von Interesse, wenn diese z. B. anderen Flugkörpern (Vögel, andere UAV s, etc.) selbsttätig ausweichen sollen. Autonome UAS könnten so in Bereichen eingesetzt werden, die eine Steuerung per Sichtkontakt und Fernsteuerung nicht zulassen - sie können sogar bei fehlenden GNSS-Signalen eingesetzt werden (Artieda et al. 2009). Mit ihnen wäre zudem eine detaillierte Aufzeichnung von Stollen und Höhlen mit geringem Risiko und Ressourceneinsatz möglich (Azhari et al. 2017). Auch in Katastrophengebieten, nach Erdbeben oder während Vulkanausbrüchen könnten solche autonomen UAS eingesetzt werden, um Menschen zu retten, Infrastrukturen zu sichern oder Informationen für Entscheider zu sammeln (Iqbal et al. 2015; Xu et al. 2014; Erdelj et al. 2017). senschaft existierenden Datenmanagement-Systeme konnten im letzten Jahrzehnt nicht mit diesen Datenmengen mitwachsen. Servercluster, die sowohl eine Speicherung wie auch den einfachen und uneingeschränkten Zugriff auf Daten zulassen, sind derzeit kaum vorhanden. Sollen zudem Anwendungen, Algorithmen oder komplexe Processing-Verfahren eingesetzt werden, sind die Serverkapazitäten in Deutschland derzeit als nicht vorhanden einzustufen. Noch gravierender ist, dass die Archivierung von Processing-Werkzeugen und damit die für den wissenschaftlichen Prozess wichtige Reproduzierbarkeit so nicht gesichert scheint (Franke et al. 2015; DFG 2013).

Mit diesen Problemen verbunden ist die Herausforderung, die neuen Datensätze mit bereits existierenden zu verknüpfen. Umweltforschung und Geowissenschaften nutzen bislang überwiegend sogenannte Rasterdaten (auch als GRID bezeichnet). Messwerte werden hier an bestimmten, meist gleichverteilten Punkten aufgenommen, was die Datenverarbeitung deutlich vereinfacht. Seit einem Jahrzehnt sind darüber hinaus z. B. Lidardaten mit einer anderen Datenstruktur im Forschungsbereich vorhanden: Punktwolken mit irregulären Abständen der Messwerte zueinander bieten verbesserte räumliche Auflösungen, erfordern aber auch neue Interpretationsroutinen (z. B. durch die Überführung in Triangulated Irregular Networks, TIN) und fundiertes Fachwissen (Perroy et al. 2010). Die Punktwolken werden zur Analyse häufig in Rasterdaten (Digitales Geländemodell) umgewandelt, um ein vereinfachtes Arbeiten zu ermöglichen (Passalacqua et al. 2010; Roering et al. 2013).

Mit UAS werden solche detaillierten Punktwolken z. B. über fotogrammetrische Verfahren aufgezeichnet. Für die Messung von Veränderungen der Erdoberfläche mit mehreren, zeitlich versetzten Aufnahmen (change detection) werden aber komplexere Analysemethoden benötigt, die eine bessere Vergleichbarkeit erlauben (Saur und Krüger 2016). Die Daten müssen hierzu auf ein einheitliches geografisches Format gebracht werden, was 
bei unterschiedlichen Flugverhalten mit z. T. großem Aufwand verbunden ist. Fliegt das UAS z. B. wegen wechselhafter Windverhältnisse nicht mit gleichmäßiger Geschwindigkeit, sind auch die Messpunkte nicht gleichmäßig verteilt. In der Verarbeitung müssen daher komplexere Algorithmen genutzt werden, die diese unterschiedlichen Punktabstände berücksichtigen. Verschiedene universitäre Arbeitsgruppen widmen sich diesem Problem. Sie versuchen, bestehende Rasterdaten mit neuen Punktwolken zu kombinieren (Perroy et al. 2010; Brell et al. 2016; Brell et al. 2017). Ziel ist es, Erdbeobachtungen, die von Satelliten oder anderen Flugobjekten aus gemacht werden, mit den Beobachtungsdaten der UAS zu verschmelzen. Dies erlaubt die Satellitendaten zu kalibrieren und großflächig präzisere Aussagen zu treffen - eine wichtige Aufgabe z. B. für den Katastrophenschutz. Um hier aber ein überregionales oder gar globales Produkt für Anwender und Entscheider zu entwickeln, müssen die angesprochenen Defizite der Serverkapazitäten behoben werden.

Gleichzeitig zeichnet sich ab, dass die neuen Möglichkeiten einer flächendeckenden dreidimensionalen Beobachtung die Begrenzungen der Serverkapazitäten noch verschärfen. Durch geschickte Routenplanung und Methodiken wie Structure-from-Motion können nun dreidimensionale Umweltdaten in höchster Auflösung erzeugt werden. Biologen vermessen z. B. mithilfe von UAS die Biomasse einzelner Bäume oder ganzer Wälder (Alonzo et al. 2014). Hierdurch wird es möglich, die Speicherkapazitäten für Kohlenstoffdioxid mit großer Genauigkeit zu bestimmen - Daten, die für Klimamodelle dringend erforderlich sind. Die Datenmengen steigen durch diese 3-D-Anwendungen enorm an. Die Visualisierung der Daten scheint dabei gelöst - ihre Speicherung und Auswertung sind jedoch nach wie vor Herausforderungen für die Wissenschaft. Anwendungen in der Versicherungswirtschaft, der Energiebranche oder in Planung und Logistik stellen dabei nur die Spitze möglicher Märkte abseits der rein wissenschaftlichen Nutzung dar.
2016). So sind z. B. über Monate hinweg Beobachtungen der Änderungen der Feinstaubbelastungen in ländlichen Räumen möglich. Insbesondere der Wechsel von punktuellen Messdaten hin zu Beobachtungen im städtischen oder ländlichen Kontext erweitert das Verständnis der Mikroklimadynamik. Langzeitbeobachtungen erzeugen aber unmittelbar größere Datenmengen. Begrenzte Kapazitäten zur Daten- und Prozessspeicherung groBer Datenmengen stellen eine Herausforderung dar, die kurzfristig angegangen werden könnte. Mittelfristig folgen daraus für die Wissenschaft jedoch Probleme bezüglich Wartung, Unterhalt oder auch hinsichtlich des Vertrauens in solche Server-Cluster (Potthoff et al. 2014). Gemeint sind damit zwar auch Allgemeinkosten, wichtiger scheint aber, dass das notwendige Personal zur Verfügung steht. Dies öffnet eine zweite Dimension der Herausforderungen für den Gebrauch von UAS in der Wissenschaft: die Ausbildung von Experten.

\section{Personalressourcen}

Nach dem geläufigen Muster werden Wissenschaftler in einem eher als autodidaktisch zu wertenden Prozess zu UAS-Piloten. Meist bereits mit dem „Drohnen-Virus“ befallen und im Hobby-Bereich aktiv, nutzen Forscherinnen und Forscher UAS auch in ihrer wissenschaftlichen Arbeit. Sie haben oft privat gesammelte Flugerfahrungen und sind mit wenigen Flugsystemen vertraut (im Hobbybereich meist kleine Quadrocopter). In der wissenschaftlichen Anwendung treffen sie nun auf Fixed-Wing-Systeme oder Multicopter mit hohen Traglasten. Eine Ausbildung oder ein Training mit solchen Systemen wird jedoch in der Regel nicht professionalisiert angeboten. Zwar bieten einige Hersteller entsprechende Schulungen im Umfeld des Kaufs von UAV-Systemen an, jedoch sind solche Schulungen bei einem bestehenden Gerätepool von mehreren unterschiedlichen Systemen kaum mehr für Forschungseinrichtungen finanzierbar. Für ältere oder modifizierte UAVs sind entsprechende Angebote in der Regel

\section{Die Visualisierung der Daten scheint gelöst- ihre Speicherung und Auswertung sind nach wie vor Herausforderungen für die Wissenschaft.}

Neben der Datenverschmelzung und der Dreidimensionalität verschärft ein weiterer Faktor den Bedarf an Serverkapazitäten: die Zeitreihenbeobachtung. War es bisher aufgrund logistischer und technischer Herausforderungen nur möglich, punktuell Umweltbeobachtungen über eine längere Zeit zu realisieren, können solche Daten mithilfe von UAS nun auch flächenhaft erzeugt werden. Wurden z. B. an fest stationierten Klimamessstationen in Städten bisher wertvolle Zeitreihen umweltrelevanter Faktoren wie Feinstaubbelastung, städtische Klimadaten oder Nutzungsmuster registriert, können diese Daten mit UAS flächendeckend und regelmäßig aufgezeichnet werden (Villa et al. nicht zu finden. Curriculare Angebote fehlen in der Regel völlig. Einige Hochschulen bieten seit neustem Flugtrainings in Sommerschulen an, vielerorts finden aber keine entsprechenden Praxis-Trainings statt. Hier ist die Wissenschaft derzeit stark auf das persönliche Engagement von Forscherinnen und Forschern angewiesen. Ein deutschlandweites Netz an Prüfungsstellen ermöglicht nun aber den räumlich nahen Zugang zum geforderten Kenntnisnachweis und damit zu einer theoretischen Ausbildung und Prüfung in Luftrecht, Meteorologie und Flugverfahren.

Eine strukturelle Unterstützung aufzubauen scheint daher Aufgabe von Hochschulen und Forschungseinrichtungen selbst 
zu sein. Im Raum Potsdam/Berlin oder auch in Göttingen haben sich daher Netzwerke der wissenschaftlichen UAS-Nutzer gebildet, in denen entsprechende Angebote institutionsübergreifend realisiert werden, z. B. Workshops zur Datenbearbeitung von Punktwolken (ausgerichtet durch UNAVCO und die International Research Training Group Strategy an der Universität Potsdam) oder zu rechtlichen Rahmenbedingung der UAS-Nutzung in der Wissenschaft (unterstützt durch das Geo.X Netzwerk).

Neben der flugpraktischen Ausbildung sind auch anwendungsbezogene Kompetenzen wichtiger Bestandteil einer Nachwuchsschulung. Grundlagen des Datenhandlings und der Datenverarbeitung sind dabei Kernaufgaben der Hochschulen.

\section{Die Wissenschaftslandschaft}

\section{in Deutschland stößt durch den}

\section{Datenzuwachs an Kapazitätsgrenzen.}

Zahlreiche Seminare und ganze Studiengänge sind um diese Kompetenzen entstanden (Helbig 2016). Deutschland hat sich hierbei in der internationalen Spitzengruppe der UAS-Datenwissenschaften etabliert. Von hohem Wert ist die Kooperation zwischen Umwelt-, Bio- und Geowissenschaften mit den Computerund Datenwissenschaften. Dabei werden zunehmend Techniken des Data-Mining und der Mustererkennung aus der Informatik in die Disziplinen der UAS-Nutzer übertragen. Der Technologie- und Wissenstransfer in Bezug auf die Datenvisualisierung ist ebenfalls angestoßen. Als noch ausstehende Herausforderung ist aber der Bereich des Datenmanagements anzusehen. Die Archivierung großer Datenmengen ist mit Fachkompetenzen zu füllen, wie sie bislang von der Wissenschaft noch nicht bereitgestellt werden. Die wenigen etablierten Datenrepositorien der Geo- und Biowissenschaften sind personell meist nur mit dem Nötigsten ausgestattet. Nur dank der großen Erfahrung und der Expertise dieser Fachleute ist der stetige Zuwachs an Daten, Datenformaten und Processing-Werkzeugen derzeit noch handhabbar. Es zeichnet sich ab, dass die Wissenschaftslandschaft Deutschlands hier an ihre Kapazitätsgrenzen stößt. Eine Ausbildung im Datenmanagement ist derzeit nur an wenigen Orten möglich (z. B. an der FH Potsdam oder der TU Dortmund, gelegentlich in Zusammenhang mit Studiengängen der Geodäsie oder Geoinformatik oder als Ausbildungsbestandteil im Vermessungswesen). Hier gilt es, den bereits absehbaren, zukünftigen Bedarf frühzeitig anzusprechen und kurzfristig entsprechende Angebote zu schaffen.

Bei der Entwicklung solcher Angebote muss aber die hohe Personalfluktuation in der Wissenschaft berücksichtigt werden. Wissenschaftler sind in Forschungsvorhaben in der Regel für wenige Jahre angestellt. Daher kommt es zu einem stetigen Austausch des Personals (Doktoranden, Postdocs, wissenschaftliche Mitarbeiter). Die angesprochene Notwendigkeit professioneller Aus- und Weiterbildung darf daher nicht nur den kurzfristigen
Bedarf decken - sie muss auch den mittel- und langfristigen Rahmenbedingungen durch entsprechende curriculare und administrative Planungen Rechnung tragen.

\section{Technologieentwicklung}

Die Nutzung von UAS in der Wissenschaft stellt spezielle Anforderungen an die Technik, die aber oft selbst Teil von Forschungsund Entwicklungsvorhaben ist. Da hierbei insbesondere zwei technische Komponenten zu berücksichtigen sind, Fluggerät und Datensensorik, sind aktuelle Entwicklungen in einem sehr breiten Themenfeld zu beobachten. Gleichwohl sind technische Herausforderungen auf der Fluggerät-Ebene insbesondere von Herstellern selbst getrieben, die sich durch den derzeit boomenden privaten Markt zu immer neuen Innovationen antreiben lassen. So ist die Anforderung transportable UAS-Einheiten zu entwickeln in den letzten Jahren bereits sehr gut umgesetzt worden. Dies ist für Anwender aus den Bio- und Geowissenschaften von großem Interesse, da hier Forschungsvorhaben in aller Regel abgelegene Regionen, meist im internationalen Kontext, betreffen. Weitere Entwicklungen sind zudem im Umfeld geräuschmindernder Konzepte, langlebiger Batteriesysteme und präziser Steuerung zu erwarten.

Sehr spezielle technische Anforderungen an UAS leiten sich aus den wissenschaftlichen Fragestellungen selbst ab. So werden Sensortechnologien genutzt, die abseits von Massenmärkten zu sehen sind (Nebiker et al. 2015). Infrarot- und Multispektralsensorik, Radar- und Laser-Technologien oder Lidar-Anwendungen verlangen nach speziellen Träger- und Bussystemen. Solche Bussysteme müssen einen einfachen und schnellen Wechsel der Sensorik oder gar eine parallele Nutzung unterschiedlicher Verfahren ermöglichen. Hier werden vielerorts individuelle Lösungen entwickelt. In letzter Zeit sind erste Universal-Systeme verfügbar geworden, die jedoch ihre Tauglichkeit im Wissenschaftsumfeld noch unter Beweis stellen müssen. Von Interesse sind auch platzsparende Lösungen, die den Einsatz kleiner, einfach zu transportierender Drohnen erlauben. Solche Drohnen sind neben der einfacheren Logistik auch sinnvoll, wenn Drohnen-Schwärme eingesetzt werden sollen. Der gleichzeitige Einsatz von mehreren, aufeinander abgestimmten UAS könnte z. B. den Einsatz hochsensibler Messverfahren erlauben, wie sie in der Prospektion denkbar sind. Bisher fehlt es neben den erwähnten platzsparenden Sensoriken zudem an Flugregelungs- und Missionssteuerungslösungen, die bei stark unsicheren Systemund Umgebungseigenschaften greifen. Derzeit wird daher geforscht, Kamera- und Laser-Systeme als Sensoren in der Flugsteuerung einzusetzen. Dies würde eine zusätzliche Sicherheit im Flugbetreib einzelner UAS ergeben und könnte die Fluglageund Positionsbestimmung in UAS-Schwärmen untereinander ermöglichen (He et al. 2018).

\section{Administrative Strukturen}

Die Frage nach Haftpflichtversicherungen, die für Aufstiegsgenehmigungen notwendig sind, ist aufseiten der Hochschulen bisher kaum gelöst. Zwar unterliegen viele Universitäten dem 
Grundsatz der Selbstversicherung, doch wird diese von vielen wissenschaftlichen Nutzern als unzureichend bemängelt. Wenn Forschende im Sinne des sicheren Betriebs von UAS ausgebildet werden sollen, so müssen auch Studierende UAS fliegen dürfen. Hier erhofft sich die forschende Seite der Hochschulen von der administrativen Ebene rasch ein Konzept. In der Tat werden bereits vielerorts intensiv Gespräche geführt. Welche Lösungen sich letztlich als realisierbar und effizient erweisen werden, bleibt aber abzuwarten.

Auf europäischer Ebene deutet sich an, dass in naher Zukunft die Zuständigkeit und damit auch die Regelungen für die unbemannte Luftfahrt vom europäischen Parlament erneuert werden. Zur Stärkung des europäischen Marktes für UAS wird die Zuständigkeit für die unbemannte Luftfahrt auch unterhalb von $150 \mathrm{~kg}$ zukünftig in die Zuständigkeit der Europäischen Agentur für Flugsicherheit (EASA) gelegt. Damit ist der Weg geebnet, dass in Deutschland und Europa einheitliche Verfahren und Regelungen Einzug halten und Hindernisse bei der raschen Entwicklung der unbemannten Luftfahrt überwunden werden. Hier geht Europa einen fälligen, mutigen und begrüßenswerten Schritt in die gemeinsame Zukunft. Die UAS-Nutzer aus der Wissenschaft hoffen, dass auch ihre Perspektive bei der Konzeption EU-weiter Vorgaben berücksichtigt wird.

\section{Anmerkung}

Diese Publikation ist im Rahmen der Forschungsinitiative NEXUS an der Universität Potsdam sowie der Initiative GeoEd - Mehr Geowissen in der Bildung (Universität Potsdam und GeoUnion Alfred-Wegener-Stiftung) entstanden. NEXUS wird vom Brandenburgischen Ministerium für Wissenschaft, Forschung und Kultur (MWFK) unterstützt.

\section{Literatur}

Alonzo, Mike; Bookhagen, Bodo; Roberts, Dar (2014): Urban tree species mapping using hyperspectral and lidar data fusion. In: Remote Sensing of Environment 148, S. 70-83.

Artieda, Jorge et al. (2009): Visual 3-D SLAM from UAVs. In: Journal of Intelligent and Robotic Systems 55, S. 229-321.

Azhari, Faris et al. (2017): A comparison of sensors for underground void mapping by unmanned aerial vehicles. In: M. Hudyma und Y. Potvin (Hg.): Underground mining technology. Sudbury, Australia: Australian Centre for Geomechanics. Online verfügbar unter https://papers.acg.uwa.edu.au/d/1710_33_ Sennersten/33_Sennersten.pdf, zuletzt geprüft am 04.10.2018.

Brell, Maximilian; Rogass, Christian; Segl, Karl; Bookhagen, Bodo; Guanter, Luis (2016): Improving sensor fusion. A parametric method for the geometric coalignment of airborne hyperspectral and lidar data. In: IEEE Transactions on Geoscience and Remote Sensing, 54 (6), S.3460-3474.

Brell, Maximilian; Segl, Karl; Guanter, Luis; Bookhagen, Bodo (2017): Hyperspectral and lidar intensity data fusion. A framework for the rigorous correction of illumination, anisotropic effects, and cross calibration. In: Transactions on Geoscience and Remote Sensing 55 (5), S. 2799-2810.

Campana, Stefano (2017): Drones in archaeology. State-of-the-art and future perspectives. In: Archaeological Prospection 24 (4), S. 275-296.

D'Andrea, Raffaello (2014): Can drones deliver? In: IEEE Transactions on Automation Science and Engineering 11 (3), S. 647-648. DOI: 10.1109/TASE.2014.2326952.
DFG - Deutsche Forschungsgemeinschaft (2013): Langzeitperspektiven und Infrastruktur der terrestrischen Forschung Deutschlands. Ein systemischer Ansatz. Online verfügbar unter http://www.dkn-future-earth.org/ data/mediapool/strategiepapier_infrastruktur_de.pdf, zuletzt geprüft am 04.10.2018.

Erdelj, Milan; Król, Michal; Natalizio, Enrico (2017): Wireless sensor networks and multi-UAV systems for natural disaster management. In: Computer Networks 124, S.72-86. DOI: 10.1016/j.comnet.2017.05.021.

Fallavollita, Paolo et al. (2013): UAS for archeology. New perspectives on aerial documentation. In: International Archives of the Photogrammetry, Remote Sensing and Spatial Information Sciences XL-1/W2, S. 131-135. DOI: 10.5194/ isprsarchives-XL-1-W2-131-2013.

Franke, Michael et al. (2015): Positionspapier „Research data at your fingertips“ der Arbeitsgruppe Forschungsdaten. Potsdam: Deutsches Geoforschungszentrum. DOI: 10.2312/allianzfd.001.

Haala, Norbert; Schwieger, Volker (2017): UAV. Anforderungen und Möglichkeiten. In: Aiham Hassan (Hg.): Unmanned Aerial Vehicles 2017 (UAV 2017). Beiträge zum 156. DVW-Seminar am 9. und 10. Februar 2017 in Stuttgart. Augsburg: Wißner-Verlag, S.9-26. Online verfügbar unter https://geodaesie.info/ system/files/privat/DVW_86_2017_UAV_2017_FINAL_170124.pdf, zuletzt geprüft am 04.10.2018.

He, Lvlong; Bai, Peng; Liang, Xialong; Zhang, Jiaqiang; Wang, Weijia (2018): Feedback formation control of UAV swarm with multiple implicit leaders. In: Aerospace Science and Technology 72, S. 327-334.

Helbig, Kerstin (2016): Research data management training for geographers. First impressions. In: ISPRS International Journal of Geo-Information 5 (4). DOI: $10.3390 / i j g i 5040040$.

Iqbal, Umair et al. (2015): Selection of unmanned aerial systems (UAS) for disaster relief operations. A comparison. In: Science International (Lahore) 27 (4), S. 3199-3203.

Korn, Bernd; Edinger, Christiane (2008): UAS in civil airspace. Demonstrating "sense and avoid" capabilities in flight trials. In: IEEE/AIAA 27th Digital Avionics Systems Conference, S. 4.D.1-1 bis 4.D.1-7.

Nebiker, Stephan; Annen, Adrian; Scherrer, Marco; Oesch, David (2008): A light-weight multispectral sensor for micro UAV. Opportunities for very high resolution airborne remote sensing. In: The International Archives of the Photogrammetry, Remote Sensing and Spatial Information Sciences 37, S. 1193-1198.

Nebiker, Stephan; Lack, Natalie; Läderach, Sonja (2015): UAV-Fernerkundung. Neue Sensoren und Anwendungen. In: Geomatik Schweiz 113, S. 351-355. DOI: $10.5169 /$ seals-513920.

Passalacqua, Paola et al. (2015): Analyzing high resolution topography for advancing the understanding of mass and energy transfer through landscapes. A review. In: Earth-Science Review 148, S. 174-193.

Perroy, Ryan; Bookhagen, Bodo; Asner, Gregory; Chadwick, Oliver (2010): Comparison of gully erosion estimates using airborne and ground-based LiDAR on Santa Cruz Island, California. In: Geomorphology 118, S. 288-300.

Potthoff, Jan; Wezel, Jos Van; Razum, Matthias; Walk, Marius (2014): Anforderungen eines nachhaltigen, disziplinübergreifenden Forschungsdaten-Repositoriums. In: Paul Müller, Bernhard Neumair, Helmut Reiser und Gabi Rodosek. (Hg.): 7. DFN-Forum - Kommunikationstechnologien. Bonn: Gesellschaft für Informatik e. V., S. 11-20.

Roering, Joshua et al. (2013): You are HERE. Connecting the dots with airborne lidar for geomorphic fieldwork. In: Geomorphology 200, S. 172-183. 
Saarinen, Ninni et al. (2018): Assessing biodiversity in boreal forests with UAV-based photogrammetric point clouds and hyperspectral imaging. In: Journal of Remote Sensing 10 (2). DOI: 10.3390/rs10020338.

Saur, Günter; Krüger, Wolfgang (2016): Change detection in UAV video mosaics combining a feature based approach and extended image differencing. In: The International Archives of the Photogrammetry, Remote Sensing and Spatial Information Sciences XLI-B7, S. 557-562. DOI: 10.5194/isprsarchives-XLI-B7-557-2016

Thiels, Cornelius A.; Aho, Johnathon; Zietlow, Scott; Jenkins, Donald (2015): Use of Unmanned Aerial Vehicles for medical product transport. In: Air Medical Journal 34 (2), S. 104-108.

Turner, Darren; Lucieer, Arko; Watson, Christopher (2012): An automated technique for generating georectified mosaics from ultra-high resolution unmanned aerial vehicle (UAV) imagery, based on structure from motion (SfM) point clouds. In: Remote Sensing 4 (5), S. 1392-1410. D0I: 10.3390/ rs4051392.

Turner, Darren; Lucieer, Arko; de Jong, Steven (2015): Time series analysis of landslide dynamics using an Unmanned Aerial Vehicle (UAV). In: Journal of Remote Sensing Vol 7 (2), S. 1736-1757. DOI: 10.3390/rs70201736.

Villa, Tommaso; Gonzalez, Felipe; Miljievic, Branka; Ristovski, Zoran; Morawska, Lidia (2016): An overview of small unmanned aerial vehicles for air quality measurements. Present applications and future prospectives. In: Sensors 16 (7). DOI: 10.3390/s16071072.

Westoby, Matthew; Brasington, James; Glasser, Neil; Hambrey, Michael; Reynolds, John (2012): ,Structure-from-Motion' photogrammetry. A low-cost, effective tool for geoscience applications. In: Geomorphology 179, S. 300-314. DOI: 10.1016/j.geomorph.2012.08.021.

Xu, Zhiqiang et al. (2014): Development of an UAS for post-earthquake disaster surveying and its application in ms7.0 Lushan earthquake, Sichuan, China. In: Computers \& Geoscience 68, S. 22-30.

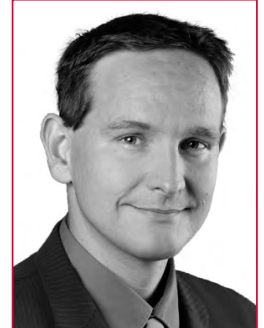

\section{DR. SIMON SCHNEIDER}

hat nach dem Diplom-Studium der Geophysik die Öffentlichkeitsarbeit eines bundesweiten Forschungsprogramms koordiniert. Seine Promotion in der Wissenschaftskommunikation befasste sich mit kulturellen und regionalen Parametern, die die Berichterstattung von Forschungsthemen in der Tagespresse beeinflussen. Er ist heute im Wissenschaftsmanagement des Forschungsschwerpunkts Erdwissenschaften der Universität Potsdam tätig.

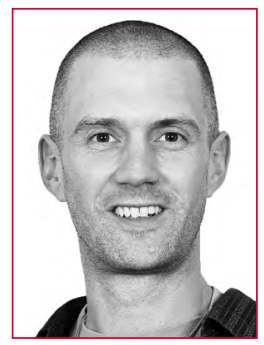

\section{PROF. DR. BODO BOOKHAGEN}

leitet den Lehrstuhl für Geologische Fernerkundung an der Universität Potsdam und beschäftigt sich mit verschiedenen Fragestellungen der Geowissenschaften im Bereich der Punktwolken, Untersuchungen von digitalen Geländemodellen sowie der Analyse von radar, radar-interferometrischen und optischen Zeitreihen. Zuvor: zehn Jahre als Professor für Erdoberflächenprozesse und Fernerkundung an der UC Santa Barbara; Postdoc-Stationen in Santa Barbara, Berkeley und Stanford; Promotion in Geologie; Diplomstudium der Geowissenschaften.

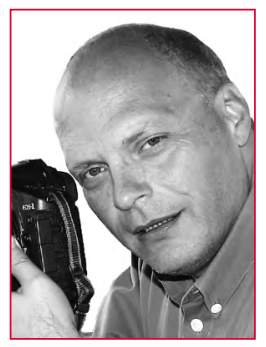

\section{PAUL ESCHBACH}

ist Ingenieur und Wirtschaftsingenieur. Er berät Industrieunternehmen und Mittelstand zu den Themen Energie und Effizienz. In der Luftbildfotografie hat er über drei Jahrzehnte eine große Expertise entwickelt. Im UAV DACH leitet er als Referent die Presse- und Öffentlichkeitsarbeit und ist seit 2016 im Verein. Eine eigene Prüfstelle für den Kenntnisnachweis bringt den fachlichen Einblick in die UAV DACH Themen.

\section{Wie Sie für die Rubriken Forschung, Reflexionen und Aus dem Netzwerk TA Beiträge einreichen}

Forschung (peer reviewed)

Hier gilt ein offener Call for Papers. Sie können Ihre Abstracts oder Manuskripte mit Bezug zum interdisziplinären Feld der Technikfolgenabschätzung jederzeit einreichen.

\section{Reflexionen}

Für diese nicht begutachtete Rubrik können Sie jederzeit Buchrezensionen, aktuelle Tagungsberichte oder andere kürzere Texte mit Bezug zum interdisziplinären Feld der Technikfolgenabschätzung einreichen.
Aus dem Netzwerk TA

Mit einer kostenpflichtigen Veröffentlichung in dieser Rubrik präsentieren Sie Ihre Institution dem breiten Rezipientenkreis von TATuP. Gleichzeitig geben Sie eine finanzielle wie ideelle Unterstützung an TATuP als Publikationsorgan der TA-Community. Informieren Sie Wissenschaft, Politik, Wirtschaft und die interessierte Öffentlichkeit z. B. über aktuelle Forschungsprojekte, Veröffentlichungen oder Stellenanzeigen an Ihrem Institut.

$100 \%$ open access! Alle Rubriken in TATuP sind kostenfrei als Druckexemplar zu beziehen beziehungsweise ohne Zugangsbeschränkung über die TATuP-Webseite zu lesen.

Weitere Informationen finden Sie unter www.tatup.de. Einreichungen schicken Sie bitte an redaktion@tatup.de. 